\title{
Modeling and Analysis of IS-IS Protocolrouting Performance Based on The Generalized Stochastic Petri Nets
}

\author{
Z.L. Liu, M.Y. Guo, T. Lan, Y. Chai, J.F. Qu \\ Chongqing University, China
}

\author{
F. Qi \\ Chongqing University, China \\ Xichang Satellite Launch Center, China
}

\begin{abstract}
Based on the analysis of basic operation process of the IS-IS(Intermediate System to Intermediate System)protocol, the paper provides a LSP (Link State Packet) diffusion model of the IS-IS protocol with the GSPN(generalized stochastic petri nets). And the simulation results show that the router's load becomes heavier significantly if the network structure's variation is greater. And also from the simulation, it is indicated that the inter-area router's load is the major load of all the routers. So the LSP(Link State Packets)diffusion timeconsuming can be reduced by improving the performance of the inter-area router in order to guarantee the fast convergence of the protocol routing computation.
\end{abstract}

Keyword-IS-IS protocol; GSPN; LSP diffusion; performance optimization

\section{INTRODUCTION}

With the extending of the network scale, the IS-IS routing protocol is widely used in many applications, as a major internal gateway protocol with layered network routing, strong scalability, fast convergence speed and good stability. So, it is significantly important for the research on the IS-IS protocol.

Currently, the research on IS-IS protocol is the focus issue of many scholars and engineers. Octavian Rusu and Valeriu Vraciu gave an optimization method of parameters which influence IS-IS protocol network performance, and provided a new route algorithm[1].Mijeong Yang and JinhoHahm designed and implemented an IS-IS protocol system of ATM machine based on multi-protocol exchange system (MPLS, Multi-protocol Label Switching), then made some testing and analysis[2]. T.Li and H.Smit made an extended research onIS-IS protocol of traffic engineering[3].K.Kompella also extended the research on ISIS protocol about generalized multi-protocol exchange system[4]. YulanZhao, etc, made a conformance tests for two types of environment, respectively are broadcast networks and peer-to-peer networks, of IS-IS Protocols based on ELOTOS[5].

Although there are a lot of researches on IS-IS Protocol, but many focused on conformance tests and design and application of IS-IS Protocol system, ignoring the analysis on the performance of the protocol router. As core component of the protocol, routers determine the protocol data processing and retransmission, and play an indispensable role in the process of the protocol operation. Strictly, the change of the network structure has been in a dynamic process, so it is necessary to carry out the router's performance analysis under the effects of the change of the network structure.

Based on the analysis of the basic operation process of the IS-IS protocol, this paper simplify the process of IS-IS protocol with the help of stochastic petri nets modeling tools and build up the LSP diffusion model of IS-IS protocol. And with the simulation of LSP diffusion time-consuming based on the stochastic petri nets when the network structure changes, this paper analyses the load of the routers and provides the reference for the performance optimization of the routers under when the network structure changes.

\section{GENERAL CONCEPTS OF THE IS-IS PROTOCOL}

\section{A. IS-IS Protocol Routing Structure}

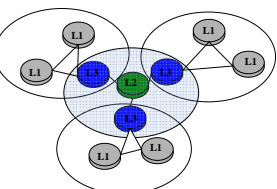

\section{FIGURE I. THE STRUCTURE OF IS-IS PROTOCOL ROUTING}

As shown in Figure 1, IS-IS protocol defines the layered area, whose border is the data link between the routers[6]. And according to the protocol, the routers are cataloged as the L1, L2 and L3, whose functions are as the followings:

Router L1: Disposes the routing information in the extraarea and only maintains the L1's LSDB(Link State Data Base)containing routing information in the area. And the packets of the above routing information, transmitted to the outside of the area, are forwarded to the nearest L3 router firstly.

Router L2: Disposes the routing information in the intraarea and only maintains the L2's LSDB containing routing information between areas. All router L2s constitute the backbone of the routing domain, which are responsible for the communication between the different areas. 
Router L3: Disposes the routing information in the interarea and maintains the linkage between L1 and L2 area. It maintains two types of LSDBs, including LSDB of L1 for extra-area routing and LSDB of L2 for intra-area routing.

\section{B. Introduction Of LSP Diffusion}

In the network, both the network protocol operations and the routing calculations depend on the LSDB synchronization. If not synchronized, there will be faults when the network operating and routing calculating. In order to get the best routing, it is necessary to launch the research on LSP diffusion which is prerequisite of link-state database synchronization.

LSP diffusion is the flooding of the link-state database for spreading routing information. LSP begins to diffuse when the adjacency connection is built. New LSP will be generated by the interface state(or data link) changes, the adjacency connection relations, the redistributions of IS-IS routing, and etc. The IS-IS routers exchange the LSP with the adjacent IS-IS routers and store all the LSPs.
There are three steps need to be done before the LSP diffusion:

Step 1: Searching the whole network LSDB of routers connecting the area.

Step2: Calculating the path and time consuming of all the nodes by the SPF (Shortest Path First) algorithm with the LSP information in LSDB.

Step3: Getting the IP routing table by the PRC (Partial Route Computing) algorithm based on the IP information reach ability in LSP. In this process, the IP information changes will not affect the network structure. That's, the ISIS protocol will run the PRC algorithm and searches the LSDB whether the network structure changes or not. But SPF's running indicates the structure changes.

Figure 2 shows the intra-area LSP diffusion, the same as that inter- and extra- area.

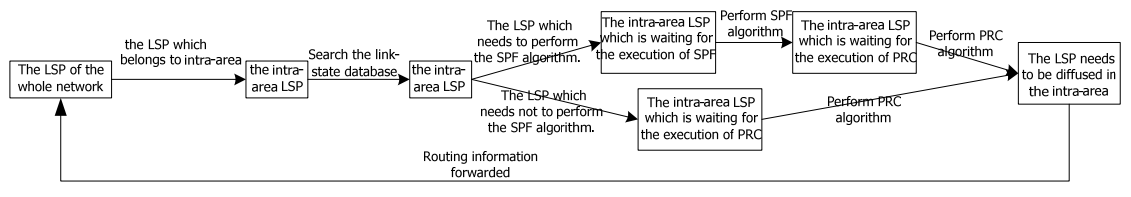

FIGURE II. THE FLOW CHART OF LSP DIFFUSION IN INTRA-AREA

\section{THE GENERALIZED STOCHASTIC PETRI NETS}

Petri nets is originated from Carl Adam Petri (Germany) doctoral thesis in 1962.As a concurrent discrete dynamic process modeling tool, petri nets has been widely applied in the modeling and performance analysis of complex systems [7-9].In order to analyze the performance of systems in the applications quantitatively, which is related to time factors, Molloy, Florin, Natkin, and etc. firstly proposed an idea that associates transitions with implementing the delay of the random index distribution[10]. There are two kinds of common stochastic petri nets: stochastic petri nets and generalized stochastic petri nets.

\section{The Definition of Generalized Stochastic Petri Nets (GSPN)}

Generalized stochastic Petri nets can be defined as a sixtuple, $\mathrm{GSPN}=(\mathrm{S}, \mathrm{T}, \mathrm{F}, \mathrm{W}, \mathrm{M}, \lambda)$, to meet the basic requirements:

(1) $\mathrm{S} \cap \mathrm{T}=\varphi$;

(2) $\mathrm{SUT} \neq \phi$;

(3) $\mathrm{F} \subseteq \mathrm{S} \times \mathrm{TUT} \times \mathrm{S}$ (" $\times$ "is Cartesian product);

(4) $\operatorname{dom}(\mathrm{F}) \cup \operatorname{cod}(\mathrm{F})=\mathrm{SUT}, \operatorname{dom}(\mathrm{F})=\{x \mid \exists y:\{x, y\} \in F\}$, $\operatorname{cod}(\mathrm{F})=\{y \mid \exists x:\{x, y\} \in F\} ;$

In the above, $\mathrm{S}$ and $\mathrm{T}$ are the place and transition sets of the GSPN.F is flow relationship. $\lambda=\left\{\lambda_{1}, \lambda_{2}, \cdots, \lambda_{k}\right\}$ isthe transition's average trigger rate[10].
As an extended stochastic Petri nets, GSPN should meet the following conditions:

(1)There can be some inhibitor arcs, which connect from places to transitions, in the flow relationship F. When one transition, connected by an inhibitor arc, is triggered, the triggering condition will be reversed. Meanwhile the tokens cannot be removed from the places connected by an inhibitor arc.

(2)The transition set includes two subsets: the time transition $T_{t}, T_{t}=\left\{t_{1}, t_{2}, \cdots, t_{k}\right\}$, and theistantaneous transition sets $T_{i}, T_{i}=\left\{t_{k+1}, t_{k+2}, \cdots, t_{n}\right\}$,where there exists $\mathrm{T}=T_{t} \cup T_{i}, T_{t} \cap T_{i}=\phi$.the transition average trigger rate $\lambda=\left\{\lambda_{1}, \lambda_{2}, \cdots, \lambda_{k}\right\}$ is associated with time transition sets, where $\lambda_{k}$ is the average implementation rate of time transition, indicating an average trigger number of times per unit time. Theinstantaneous transition sets $t_{i} \in T$ act as multiple trigger to define a random switch under a mark $M$, determining trigger probability, so it can be used as a probability transition.

\section{THE GSPN MODELING FOR LSP DIFFUSION}

Due to the network state is variable, it is necessary to synchronize link-state database by the LSP diffusion constantly. When protocol running reaches the steady state, the time consuming of the above diffusion occupies most of the protocol running time. And this process is the major part of the router's load. So LSP diffusion modeling and analysis are great significant for the router's performance evaluation and optimization. 
As shown in Figure 3, the LSP diffusion includes 3 parts: the intra-area, the inter-area and extra-area diffusion. And the intra-area LSP diffusion is as following:

Firstly, selecting the LSP only belongs to the intra-area though a probability transition $\mathrm{t} 1$. And this intra-area LSP, which is waiting for router CPU to search LSDB, namely execute transition Tr1. And the transition $\mathrm{t} 4$ and $\mathrm{t} 5$ determine whether the intra-area LSP run SPF algorithm(represented by the transition $\operatorname{Tr} 4$ ) or not. The $t 4$ is the probability transition of running SPF algorithm, whose value indicates the frequency of the network structure change. The $t 5$ is the probability transition of not running SPF algorithm, which constructs a pair of complementary probability transition with $\mathrm{t} 4$.

Secondly, the intra-area LSP will run the PRC algorithm. So it will execute transition $\operatorname{Tr} 7, \operatorname{Tr} 8$ in Figure 3.

Finally, the LSP starts to diffuse. So it will execute transition Tr13 in Figure 3.

And similar to the model of the intra-area LSP diffusion, Figure 3 shows the GSPN model for the LSP diffusion in whole networks as Figure 1.

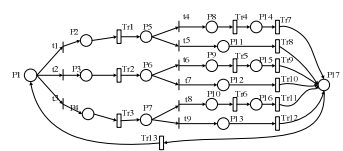

FIGURE III. THE GSPN MODELING FOR LSP DIFFUSION IN THE WHOLE NETWORKS

Table1 and Table2 show the meanings of the corresponding place and transition in Figure 3 respectively.

\begin{tabular}{|c|c|}
\hline Place & Place meaning \\
\hline P1 & The LSP of the whole network \\
\hline P2、P5 & the intra-area LSP \\
\hline P3、P6 & the inter-area LSP \\
\hline P4、P7 & the extra-area LSP \\
\hline P8 & $\begin{array}{l}\text { The intra-area LSP which is waiting for the } \\
\text { execution of SPF }\end{array}$ \\
\hline P9 & $\begin{array}{l}\text { The inter-area LSP which is waiting for the } \\
\text { execution of SPF }\end{array}$ \\
\hline P10 & $\begin{array}{l}\text { The extra-area LSP which is waiting for the } \\
\text { execution of SPF }\end{array}$ \\
\hline P11、P14 & $\begin{array}{l}\text { The intra-area LSP which is waiting for the } \\
\text { execution of PRC }\end{array}$ \\
\hline P12、P15 & $\begin{array}{l}\text { The inter-area LSP which is waiting for the } \\
\text { execution of PRC }\end{array}$ \\
\hline P13、P16 & $\begin{array}{l}\text { The extra-area LSP which is waiting for the } \\
\text { execution of PRC }\end{array}$ \\
\hline P17 & $\begin{array}{l}\text { The LSP needs to be diffused in the } \\
\text { network }\end{array}$ \\
\hline
\end{tabular}

Table II. ThE MEANING OF THE TRANSITIONS IN FIGURE 3

\begin{tabular}{|c|c|c|c|}
\hline Transition & Transition meaning & Transition & Transition meaning \\
\hline t1 & $\begin{array}{l}\text { The probability transition } \\
\text { belongs to the intra-area LSP }\end{array}$ & Tr1、Tr2、Tr3 & $\begin{array}{l}\text { Search the link-state } \\
\text { database }\end{array}$ \\
\hline $\mathrm{t} 2$ & $\begin{array}{l}\text { The probability transition } \\
\text { belongs to the inter-area LSP }\end{array}$ & Tr4、Tr5、Tr6 & $\begin{array}{l}\text { Perform SPF } \\
\text { algorithm }\end{array}$ \\
\hline $\mathrm{t} 3$ & $\begin{array}{l}\text { The probability transition } \\
\text { belongs to the extra-area LSP }\end{array}$ & $\begin{array}{l}\text { Tr7、Tr8、Tr9、 } \\
\text { Tr10、Tr11、Tr12 }\end{array}$ & $\begin{array}{l}\text { Perform PRC } \\
\text { algorithm }\end{array}$ \\
\hline $\mathrm{t} 4 、 \mathrm{t} 6 、 \mathrm{t} 8$ & $\begin{array}{l}\text { The probability transition to perform } \\
\text { SPF algorithm, the more its value } \\
\text { shows that the more frequently the } \\
\text { current network structure change. }\end{array}$ & Tr13 & $\begin{array}{l}\text { Routing information } \\
\text { forwarded }\end{array}$ \\
\hline $\mathrm{t} 5 、 \mathrm{t} 7 、 \mathrm{t} 9$ & $\begin{array}{l}\text { The probability transition not to } \\
\text { perform SPF algorithm }\end{array}$ & & \\
\hline \multicolumn{4}{|c|}{$\begin{array}{l}\text { Added: } t 1, t 2 \text { and } t 3 \text { are a pair of complementary probability transition, namely probability sum is } 1 . T 4 \\
\text { and } t 5, t 6 a n d t 7, t 8 \text { and } t 9 \text { are complementary probability transition. Namely the probability sum all are } 1 .\end{array}$} \\
\hline
\end{tabular}

\section{TIME-CONSUMING ANALYSIS OF LSP DIFFUSION WITH GSPN}

\section{A. The Time-Consuming of LSP Diffusion}

This chapter will carry out time-consuming analysis of the LSP diffusion with the GSPN constructed in the previous chapter and provide router optimization advices when the network structure changes.

After router starts IS-IS protocol, the router establishes the adjacency connection relations by sending and listening to the Hello packets periodically. Then LSP starts to diffuse after establishing the adjacency connection relations. In this paper, we consider that the routing load is resulted from the whole LSP diffusion process and the router load is consisted of the following four processes:

(1) Searching the link database: searching the LSP, represented by the transition $\operatorname{Tr} 1, \operatorname{Tr} 2$ and $\operatorname{Tr} 3$ in Figure 3. And its time-consuming complexity is $\mathrm{O}(\mathrm{m})$, supposing that $\mathrm{m}$ is an expression of the local link database's capacity.

(2) Running SPF algorithm: computing all the network nodes path and overhead, represented by $\operatorname{Tr} 4, \operatorname{Tr} 5, \operatorname{Tr} 6$ in Figure 3. And its time-consuming relates to the number of the nodes and routers in the area.

(3) Running RPC algorithm: searching the optimal path, represented by $\operatorname{Tr} 7, \operatorname{Tr} 8, \operatorname{Tr} 9, \operatorname{Tr} 10, \operatorname{Tr} 11$ and $\operatorname{Tr} 12$ in Figure 3. Since the SPF has calculated all paths in the area, the only task for running PRC to do is comparing all paths and selecting the shortest one. And it can be ignored comparing with the above two processes. So Tr7, Tr8, Tr9, Tr10, Tr11 and $\operatorname{Tr} 12$ can be viewed as transient transitions.

(4)Relaying the routing information: forwarding the current routing information to the entire network, represented by $\operatorname{Tr} 13$ in Figure 3 . The time-consuming relates to the LSP number in the area which the router connects.

\section{B. Transition Parameter Settings of LSP Diffusion Model}

With the analysis in the section 4.1 , this section will set the transition parameters of the model in the Figure 3 for quantity analysis.

Suppose that the network in Figure 1 has 3000 nodes, divided into 3 regions, each of which contains 1000 nodes. And the core router L2 of this network connects the three regions as the Figure 1. That's, L2 connects the network's 3000 nodes. 
Assuming that the link data are stored in the binary tree structure, the time complexity of searching binary tree is $\mathrm{O}$ (n), where $\mathrm{n}$ is the number of network nodes or LSPs. Since the number of LSPs is proportional to the number of nodes directly, the time complexity of searching the whole link state database is $\mathrm{O}(3000)$. And set the 1 unit rate corresponding to the time complexity of searching the whole link state database, corresponding to O (3000) here.

So the transition $\operatorname{Tr} 1, \operatorname{Tr} 2$ and $\operatorname{Tr} 3$ 's processing rates are 1 unit rate in Figure 3. Since the $\operatorname{Tr} 4, \operatorname{Tr} 5$, Tr6 processing rates are related to both the number of LSP area connected by router and that of routers in the area, the Tr4's time complexity is $\mathrm{O}$ (3000), whose corresponding transition rate is 1 unit rate. As shown in Figure 1, the whole area contains 1 intra-area router L2, 3 inter-area routers marked by L3 and 6 extra-area routers marked by L1. So the Tr5's rate is 3 times as that of the Tr4's.And the Tr6's rate is 6 times as that of the Tr4's.

The transition $\operatorname{Tr} 7, \operatorname{Tr} 8, \operatorname{Tr} 9, \operatorname{Tr} 10, \operatorname{Tr} 11$ and $\operatorname{Tr} 12$ can be viewed as an instantaneous transition, so the processing time can be ignored and the rates are infinity. Since the Tr13's time-consuming is related to the number of the whole area's LSPs, its time complexity also is O (3000). So the Tr13's rate is 1 unit rate.

And the sum of probability of intra-area router, the interarea router and extra-area routers is 1 , because the whole LSPs is composed by the LSPs of the intra-area router, the inter-area router and the extra-area routers.

Figure 1 shows that the extra-area's LSP is sent by the intra-area routers. And 1 intra-area router connects 3 interarea ones, so the number of the intra-area LSPs is 3 times as that of the inter-area LSPs. Since the transmission of extraarea LSP needs inter-area router's relaying, the number of the inter-area LSPs is the same as that of extra-area LSPs. So the ratio of the LSPs number in intra-area, inter-area and extra-area is $3: 1: 1$. And the value of $t 1, t 2, t 3$ is $0.6,0.2$ and 0.2 .Table 3 shows the parameter settings.

Table III. THEPARAMETER SETTINGS OF EACH TRANSITION IN FIGURE 3

\begin{tabular}{|c|c|c|c|}
\hline Probability transition & Probability value & Rate transition & Rate value (unit rate) \\
\hline t1 & 0.6 & $\mathrm{Tr} 1, \operatorname{Tr} 2, \operatorname{Tr} 3, \operatorname{Tr} 4, \operatorname{Tr} 1$ & 1 \\
\hline $\mathrm{t} 2$ & 0.2 & Tra & 3 \\
\hline \multirow[t]{2}{*}{$t_{3}$} & 0.2 & $\operatorname{Tr} 6$ & 6 \\
\hline & & $\begin{array}{l}\operatorname{Tr} 7, \operatorname{Tr} 8, \operatorname{Tr} 9, \\
\text { Tr10, Tr11, Tr12 }\end{array}$ & nfinity (disregard execution time) \\
\hline
\end{tabular}

\section{The Time-Consuming Analysis of LSP Diffusion Simulation}

The paper uses petri nets simulation tool PIPEv4.1 to analyzing the influence to router's performance. The simulation results mainly reflect the transitions' running, which is related to the token number in the place P8, P9, P10 and P17. And the token number reflects the load of the router when it is processing LSP diffusion. The more the token number is, the longer it takes to process the LSP Diffusion. So the paper computes routers' load under different network conditions mainly by checking the token numbers of $\mathrm{P} 8, \mathrm{P} 9$, P10 and P17.

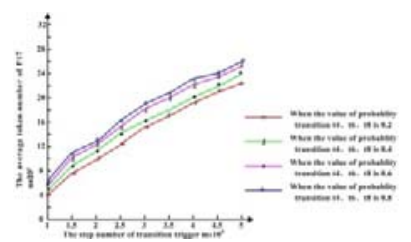

(a)the different values of probability transition $t 4 、 t 6 、 t 8$, the curve of token number in P17

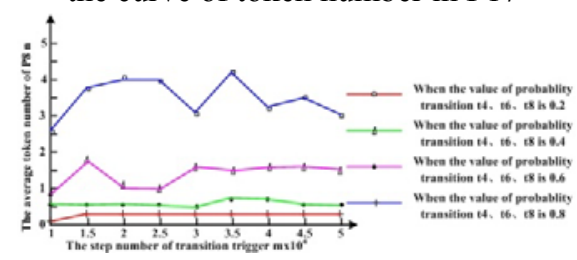

(b)the different values of probability transition 44 t 6 、 $t 8$, the curve of token number in P8

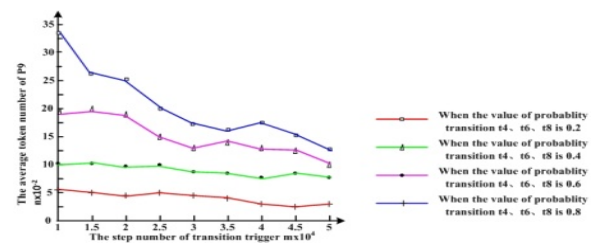

(c)the different values of probability transition 44 t6、t8, the curve of token number in P9

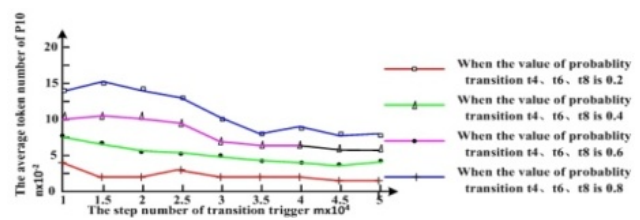

(d) the different values of probability transition $t 4 、 t 6 、 t 8$, the curve of token number in P10

FIGURE IV. THE AVERAGE TOKEN NUMBER UNDER THE DIFFERENT NETWORK STRUCTURE

In the Figure 4, the LSP diffusion time is the product of the steps of transition and the unit transition time.

As Figure 4 (a), the number of tokens in P17 will change if the network structure changes in LSP diffusion. And the more the token number is, the more frequently the network structure changes. This indicates the LSP time-consuming of the network will increase when the structure changes frequently.

Comparing with Figure 4 (b), 4 (c) and 4 (d), it can be seen that if network structure changes more sharply, the average token numbers of the corresponding place P8, P9 and P10 are larger, indicating that the routing time-consuming will 
increase. And the load will become heavier under the same circumstance.

From Figure 4 (b), 4 (c) and 4 (d), under the same network structure changing level, the relation of average token number of P8、P9 and P10 is: $\mathrm{P} 8>>\mathrm{P} 9>\mathrm{P} 10$. And under the different changing level, the average token number of P8 becomes bigger significantly with the changing level increasing. It indicates that the time-consuming of the router L2 is more than those of L1 and L3, whose load is the heaviest load of the three. And the router L2's performance mainly decides the time-consuming of LSP. So when the network changes frequently, we can improve the performance of router L2 to reduce LSP diffusion timeconsuming.

\section{CONCLUSION}

For the analysis of the IS-IS protocol and LSP diffusion, this paper employs the GSPN to model the LSP diffusion with the simulation tool PIPEv4.1.

The simulation results show that when the network changes frequently, the time consuming of LSP diffusion and routing computation will increase. The results also indicate that the routers' load become heavier when the network structure changes greater, among which the core router, L2 in Figure 1, has the heaviest load, whose performance is the major factors of LSP diffusion. So, when the network changes frequently, LSP diffusion time can be reduced by improving the performance of the core router.

\section{REFERENCES}

[1] Octavian Rusu, ValeriuVraciu, IS-IS metric optimization, Proc. of the 9thRoedunet International Conference, eds. R. Brad, IEEE: Sibiu, pp.282-286, 2010

[2] Mijeong Yang, JinhoHahmetal,Design and Implementation of the ISIS Routing Protocolwith Traffic Engineering, Proc. ofthe 9th AsiaPacific Conference on Communications, eds. M. Azhar\&A. Anuar,IEEE: Penang,pp.1102-1105, 2003

[3] T.Li, H.Smit, IS-ISextensions for Traffic Engineering, http://tools.ietf.org/html/rfc3784

[4] K.KompellaEd., IS-IS extensions in Support of Generalized MPLS, https://tools.ietf.org/html/rfc4205

[5] Yulan Zhao, Yingze Liu etal, Conformance Testing for IS-IS Protocol Based on E-LOTOS, Proc. of 2010 Information Theory and Information Security, eds. Y. C. Guo, Q. Luo, et al, IEEE: Beijing, pp.54-57,2010

[6] Abe Martey,CCIE. IS-IS network design solution, People's Posts and Telecommunications Press:Beijing, pp.33-34, 2002

[7] ChuangLin, Stochastic Petri Net and Network PerformanceEvaluation, Tsinghua University Press: Beijing, pp. 1027,2001

[8] Lin C., Stochastic PetriNets and System Performance Evaluation, Tsinghua University Press: Beijing, pp.1-137, 2005

[9] Osama S.Youness, Wail S. ElKilani et al,A behavior and delay equivalent petri net modelforperformance evaluation of communication protocols, Computer Communications,31(10), pp.2210-2230, 2008

[10] Huang Lin, Stochastic Petri nets and system performance evaluation,Tsinghua University Press: Beijing, pp. 19-45, 2005 